\title{
Transient heat conduction analysis of 3D solids with fiber inclusions using the boundary element method
}

\author{
F. Ma, J. Chatterjee, D. P. Henry and P. K. Banerjee*, $\dagger$ \\ Department of Civil Engineering, State University of New York at Buffalo, 240 Ketter Hall, \\ Buffalo, NY 14260, U.S.A.
}

\begin{abstract}
SUMMARY
A boundary element formulation is presented in this work for transient heat conduction analysis of threedimensional (3D) fiber-reinforced materials. The cylindrical-shaped fibers in a 3D matrix are idealized by a system of curvilinear line elements with a prescribed diameter. The variations in the temperature and flux fields in the circumferential direction are represented in terms of a trigonometric shape function together with a linear or quadratic variation in the longitudinal direction. This approach significantly reduces the modeling effort and the computing cost. The storage requirement for the convolution integrals is eliminated by adopting an accurate integration-based convolution algorithm for the surface of the hole and the fibers as well as a fast convolution algorithm for the outer boundary recently developed by the present authors.

Numerical examples are presented to demonstrate the accuracy and applicability of the proposed method of analysis of fiber-reinforced materials. Copyright (C) 2007 John Wiley \& Sons, Ltd.
\end{abstract}

Received 13 June 2006; Revised 26 March 2007; Accepted 7 May 2007

KEY WORDS: BEM; heat conduction; fiber reinforcement; shape function; convolution

\section{INTRODUCTION}

The conduction of heat in a composite is a complex phenomenon and can be understood only via a proper micromechanical analysis. Moreover, the analysis of heat flow in a transient state is inherently more complicated than a steady-state analysis. A conventional multi-region approach using a suitable numerical method requires the use of very large amount of data preparation effort, and, therefore, is not very practical for an analysis that requires repeated changes in the fiber arrangement. Thus, it is evident that, for a proper micromechanical analysis of composites, one needs to use a numerical method that is capable of efficiently idealizing the individual fibers or

\footnotetext{
*Correspondence to: P. K. Banerjee, Department of Civil Engineering, State University of New York at Buffalo, 240 Ketter Hall, Buffalo, NY 14260, U.S.A.

†E-mail: pkb@eng.buffalo.edu
} 
individual bundle of fibers, or even a woven system of fibers embedded within a three-dimensional (3D) matrix without using a large amount of manual data preparation effort. Also, the analysis must be sophisticated enough to take into account high-temperature gradients resulting from diffusion of temperature from the fiber to the matrix and allow for the interaction between the fibers through the matrix, which is absent in most commonly used theory of mixtures.

The boundary element method (BEM) is particularly suitable for the above task. The reduction of dimensionality of the problem using BEM enables very efficient modeling and analysis of fiberreinforced composites. Modeling holes and inclusions by BEM have always been very attractive because the main boundary element mesh is independent of the meshes needed for holes and inclusions. Banerjee and Butterfield [1] in 1971 first made use of this advantage in their modeling of the elastostatic pile-cap-pile-group interaction study involving 25 cylindrical piles embedded in a half space using a simplified BEM formulation. The same idea was also used by Barone and Caulk [2] in their study of steady-state heat transfer problems in molds having thin cooling holes, which was subsequently extended to 3D problems of transient heat transfer by Dargush and Banerjee [3]. All of the above work assumed a constant variation of functional behavior in the circumferential direction together with constant, linear or quadratic variation in the longitudinal directions.

Often the inclusions in a 3D body often have regular (such as cylindrical, elliptical or spherical) geometries. For such cases, one can efficiently make use of a Fourier series expansion of the unknown together with an analytical treatment of the surface geometry. After Rizzo and Shippy [4] first developed the BEM analysis of a general axisymmetric body, Wang and Banerjee [5] observed that using symmetric and anti-symmetric modes, the total work could be substantially reduced without any loss of generality. This fact was utilized by Henry and Banerjee [6] and Banerjee and Henry [7] in developing elastic analyses of solids with a large number of cylindrical holes and inclusions of small diameters, in which a new shape function in the cylindrical coordinate system using a Fourier expansion in the circumferential direction and a quadratic variation in the longitudinal direction was developed. Some of these developments form the subsequent basis of the die-cast work described by Barone and Caulk [8].

A thorough investigation of a full 3D modeling of holes and full multi-region modeling of inclusions was undertaken by Henry and Banerjee [6] as well as Banerjee and Henry [7]. They observed that, for holes and inclusions smaller than $10 \%$ of the least dimension of the region, three-term expansion of the Fourier series is required to cover all possible modes of deformation in an elastic analysis with a maximum error of less than $1 \%$. It is expected that, for heat transfer, one would get less errors than those observed for elastostatics.

Rigid or insulated holes of arbitrary shape by a full surface modeling of the hole surface was considered, respectively, by Liu et al. [9] for 3D elastic bodies and by Nishimura and Liu [10] for steady-state heat transfer problems. Additionally, they described the solution of a very large singleregion BEM system of equations using an iterative solver based on the fast multi-pole method. Such analyses of course, while impressive, cannot be applied to composites without allowing the inclusions to deform and conduct heat through them. It is also difficult to model the circumference of a very small diameter inclusion with regular boundary element because one needs to use at least eight elements in the circumferential direction to account for the curvature. Most importantly, the full multi-region surface modeling can consume significant data preparation effort, especially if the inclusions are curvilinear in the longitudinal direction or if a new geometric disposition of the inclusion is required for a rapid re-analysis. For these reasons, Banerjee et al. [11] developed a 3D composite modeling system (BESTCMS-National Aeronautics and Space Administration) 
based on BEM in which they addressed the modeling problem of fiber composites by using the analytical treatment of geometry for assemblage elements having three circumferential nodes and three longitudinal nodes. In their implementation, they assumed the Poisson ratio of the fiber and the matrix to be the same, which helped them to cancel several terms during the assembly of the fiber with the matrix.

The boundary element formulation adopted in this work for the transient heat conduction analysis contains convolution integrals which must be integrated in time as well as in space. Although there are other techniques, such as the volume integral conversion method [12] or the particular integrals method $[13,14]$, available to eliminate the need for volume integrals normally associated with direct application of the BEM to transient problems, the convolution method has been found to have superior accuracy over other available methods. The convolution method using the timedomain fundamental solution and a time-stepping technique was first employed by Tomlin [15], Chang et al. [16] and Banerjee et al. [17]. Subsequently, over the last 25 years, the convolution method has been comprehensively developed for multi-region two-dimensional (2D), axisymmetric as well as 3D transient heat conduction [3,18-20], and also for the more difficult problems of melting and solidification [21,22]. While one usually obtains excellent results using the above convolution method, this type of approach suffers from the need to store the coefficients related to temperature and flux history of the solution for evaluating the convolution integrals requiring a large amount of computer disk space and, therefore, is not suitable for solving relatively larger size problems. In addition, the applied time-dependent arbitrary variation of loading cannot be easily accommodated with this algorithm.

In this paper, a BEM formulation for the transient heat conduction analysis of fiber-reinforced composites has been presented based on the special line element technique mentioned earlier for modeling cylindrical holes and fibers $[6,7]$ of small diameters. To overcome the bottleneck arising due to storage convolution, recently developed efficient time convolution schemes [23,24] have been incorporated in the current work. The accuracy of the numerical results obtained using the proposed method of analysis is assessed by comparing them with those obtained using multi-region BEM. Good agreement between the two results clearly indicates that the methodology developed in this work has the promise to be scaled up to tackle large-scale practical problems of sufficient interest. To demonstrate the application and usefulness of such a transient algorithm for fiberreinforced materials, the developed analysis is then applied to study the transient heat conduction in a turbine blade.

\section{TRANSIENT BOUNDARY ELEMENT FORMULATION}

\subsection{Boundary integral equation representation}

Following the usual procedures in BEM for studying 3D transient heat conduction [3, 18-20], the temperature $T$ at a point $\xi$ in a composite matrix with hole can be written as

$$
\begin{aligned}
C(\xi) T(\xi, t)= & \int_{0}^{t}\left\{\int_{S}\left[g_{\mathrm{O}}(x, t, \xi, \tau) q_{\mathrm{O}}(x, \tau)-f_{\mathrm{O}}(x, t, \xi, \tau) T_{\mathrm{O}}(x, \tau)\right] \mathrm{d} S(x)\right\} \mathrm{d} \tau \\
& +\sum_{m=1}^{M} \int_{0}^{t}\left\{\int_{S^{m}}\left[g_{\mathrm{H}}(x, t, \xi, \tau) q_{\mathrm{H}}(x, \tau)-f_{\mathrm{H}}(x, t, \xi, \tau) T_{\mathrm{H}}(x, \tau)\right] \mathrm{d} S^{m}(x)\right\} \mathrm{d} \tau
\end{aligned}
$$


where $T$ and $q$ are temperature and flux, respectively. The transient kernel functions $g$ and $f$ are the components of the fundamental solutions of the governing equations due to an instant transient point heat source in an infinite solid $[18,20]$.

$C(\xi)$ are constants determined by the geometry at point $\xi . S, S^{m}$ are the surfaces of the outer boundary of the matrix and the $m$ th hole (will be used later for fiber). $M$ is the number of holes present in the body. The subscripts $\mathrm{O}$ and $\mathrm{H}$ denote the quantities on the outer surface of the matrix and the quantities on the surface of the hole, respectively.

Similarly, the boundary integral equation for the $m$ th fiber can be written as

$$
C_{\mathrm{F}}(\xi) T(\xi, t)=\int_{0}^{t}\left\{\int_{S}\left[g_{\mathrm{F}}(x, t, \xi, \tau) q_{\mathrm{F}}(x, \tau)-f_{\mathrm{F}}(x, t, \xi, \tau) T_{\mathrm{F}}(x, \tau)\right] \mathrm{d} S^{m}(x)\right\} \mathrm{d} \tau
$$

where $C_{\mathrm{F}}(\xi)$ are constants determined by the geometry at $\xi$ in fiber $m$ and $T_{\mathrm{F}}, q_{\mathrm{F}}$ are temperatures and fluxes, respectively, associated with the $m$ th fiber as explained before. $S^{m}$ is the surface of the $m$ th fiber. The subscript $\mathrm{F}$ is used to denote the quantities on the surface of the fiber.

\subsection{Temporal discretization}

Consider first the time integrals in Equations (1) and (2) as convolutions. Then, the time intervals from zero to $t$ can be divided into $N$ increments of duration $\Delta t$.

Therefore, the convolution integrals in Equations (1) and (2) with limits from 0 to $t$ can be expressed as

$$
\int_{0}^{t} g(x, t, \xi, \tau) q(x, \tau) \mathrm{d} \tau=\sum_{n=1}^{N} \int_{(n-1) \Delta t}^{n \Delta t} g(x, t, \xi, \tau) q(x, \tau) \mathrm{d} \tau
$$

and

$$
\int_{0}^{t} f(x, t, \xi, \tau) T(x, \tau) \mathrm{d} \tau=\sum_{n=1}^{N} \int_{(n-1) \Delta t}^{n \Delta t} f(x, t, \xi, \tau) T(x, \tau) \mathrm{d} \tau
$$

At this point, if the temporal variations of the field variables are assumed to remain constant within each time increment $\Delta t$, these variables can be brought outside the integrals appearing in Equations (3) and (4) and the convolution integrals become

$$
\int_{0}^{t} g(x, t, \xi, \tau) q(x) \mathrm{d} \tau=\sum_{n=1}^{N} q^{n}(x) \int_{(n-1) \Delta t}^{n \Delta t} g(x, t, \xi, \tau) \mathrm{d} \tau
$$

and

$$
\int_{0}^{t} f(x, t, \xi, \tau) T(x) \mathrm{d} \tau=\sum_{n=1}^{N} T^{n}(x) \int_{(n-1) \Delta t}^{n \Delta t} f(x, t, \xi, \tau) \mathrm{d} \tau
$$

The time integrals appearing in Equations (5) and (6) are evaluated analytically and the resulting transient boundary kernels were developed by Dargush and Banerjee [3] and Dargush [18]. They are also listed in the Appendix. 
The temporally discretized boundary integral equations for the $N$ th time step can now be derived. For the composite matrix with hole,

$$
\begin{aligned}
C T^{N}(\xi, t)= & \sum_{n=1}^{N}\left\{\int_{S}\left[G_{\mathrm{O}}^{n}(x, t, \xi) q_{\mathrm{O}}^{n}(x)-F_{\mathrm{O}}^{n}(x, t, \xi) T_{\mathrm{O}}^{n}(x)\right] \mathrm{d} S(x)\right. \\
& \left.+\sum_{m=1}^{M} \int_{S^{m}}\left[G_{\mathrm{H}}^{O}(x, t, \xi) q_{\mathrm{H}}^{n}(x)-F_{\mathrm{H}}^{n}(x, t, \xi) T_{\mathrm{H}}^{n}(x)\right] \mathrm{d} S^{m}(x)\right\}
\end{aligned}
$$

For the $m$ th fiber,

$$
C_{\mathrm{F}} T^{N}(\xi, t)=\sum_{n=1}^{N} \int_{S^{m}}\left[G_{\mathrm{F}}^{n}(x, t, \xi) q_{\mathrm{F}}^{n}(x)-F_{\mathrm{F}}^{n}(x, t, \xi) T_{\mathrm{F}}^{n}(x)\right] \mathrm{d} S^{m}(x)
$$

The summations on the right-hand sides of Equations (7) and (8) can be separated into two parts, one for the leading time step and the other indicating the convolution due to past events.

Thus, for the composite matrix with hole, we can write

$$
\begin{aligned}
C T^{N}(\xi, t)= & \int_{S}\left[G_{\mathrm{O}}^{1}(x, t, \xi) q_{\mathrm{O}}^{N}(x)-F_{\mathrm{O}}^{1}(x, t, \xi) T_{\mathrm{O}}^{N}(x)\right] \mathrm{d} S(x) \\
& +\sum_{m=1}^{M} \int_{S^{m}}\left[G_{\mathrm{H}}^{1}(x, t, \xi) q_{\mathrm{H}}^{N}(x)-F_{\mathrm{H}}^{1}(x, t, \xi) T_{\mathrm{H}}^{N}(x)\right] \mathrm{d} S^{m}(x)+R_{\mathrm{OH}}^{n-1}(\xi, t)
\end{aligned}
$$

where $R_{\mathrm{OH}}^{N-1}(\xi, t)$ is the summation due to past convolution due to the outer boundary and the surface of the hole given by

$$
\begin{aligned}
R_{\mathrm{OH}}^{N-1}(\xi, t)= & \sum_{n=1}^{N-1}\left\{\int_{S}\left[G_{\mathrm{O}}^{n}(x, t, \xi) q_{\mathrm{O}}^{n}(x)-F_{\mathrm{O}}^{n}(x, t, \xi) T_{\mathrm{O}}^{n}(x)\right] \mathrm{d} S(x)\right. \\
& \left.+\sum_{m=1}^{M} \int_{S^{m}}\left[G_{\mathrm{H}}^{n}(x, t, \xi) q_{\mathrm{H}}^{n}(x)-F_{\mathrm{H}}^{n}(x, t, \xi) T_{\mathrm{H}}^{n}(x)\right] \mathrm{d} S^{m}(x)\right\}
\end{aligned}
$$

Note, for the first time step $(n=1), R_{\mathrm{OH}}^{n-1}(\xi, t)=0$, that is, there is no convolution.

Similarly, for the $m$ th fiber, we can write

$$
C_{\mathrm{F}} T^{N}(\xi, t)=\int_{S^{m}}\left[G_{\mathrm{F}}^{1}(x, t, \xi) q_{\mathrm{F}}^{N}(x)-F_{\mathrm{F}}^{1}(x, t, \xi) T_{\mathrm{F}}^{N}(x)\right] \mathrm{d} S^{m}(x)+R_{\mathrm{F}}^{N-1}(\xi, t)
$$

where $R_{\mathrm{F}}^{N-1}(\xi, t)$ is the summation due to past convolution due to the fiber, given by

$$
R_{\mathrm{F}}^{N-1}(\xi, t)=\sum_{n=1}^{N-1}\left\{\int_{S^{m}}\left[G_{\mathrm{F}}^{n}(x, t, \xi) q_{\mathrm{F}}^{n}(x)-F_{\mathrm{F}}^{n}(x, t, \xi) T_{\mathrm{F}}^{n}(x)\right] \mathrm{d} S^{m}(x)\right\}
$$




\subsection{Spatial discretizations}

Next, a spatial discretization is necessary to permit the evaluation of the surface integrals appearing in the temporally discretized boundary integral equations for the composite matrix with hole and those of the fibers.

For the outer boundary of the composite matrix with hole, following the usual procedure [20], by sub-dividing the surface of the body into individual elements and invoking appropriate shape functions, the above spatial discretization can be accomplished. Once the discretization is defined, the nodal quantities can be brought outside the surface integral and the remaining shape functions and kernel products can be integrated.

However, for the surface of the fibers, a circular shape function [7] is employed to approximate the variation in the temperatures and fluxes about the circumference of the fiber/hole using three nodes generated internally by the program around the user input nodes along the centerline of the fibers (see Figure 1). The circular shape function is multiplied and integrated with the 3D transient kernels, so that the nodal values of variables can be brought outside the integral. Using the shape functions mentioned above, the temperature and flux can be expressed as

$$
T=M^{\gamma} T^{\gamma}, \quad q=M^{\gamma} q^{\gamma}
$$

In the above expressions $T^{\gamma}$ are nodal values of temperatures and $q^{\gamma}$ are nodal values of fluxes, respectively. The summation over $\gamma$ is implied, $\gamma=1,2,3$ for the three circumferential nodes as depicted in Figure 2.

The circular shape functions described in Figure 2 can be expressed as

$$
\begin{aligned}
& M^{1}(\theta)=\frac{1}{3}+\frac{2}{3} \cos \theta \\
& M^{2}(\theta)=\frac{1}{3}+\frac{\sqrt{3}}{3} \sin \theta-\frac{1}{3} \cos \theta \\
& M^{3}(\theta)=\frac{1}{3}-\frac{\sqrt{3}}{3} \sin \theta-\frac{1}{3} \cos \theta
\end{aligned}
$$

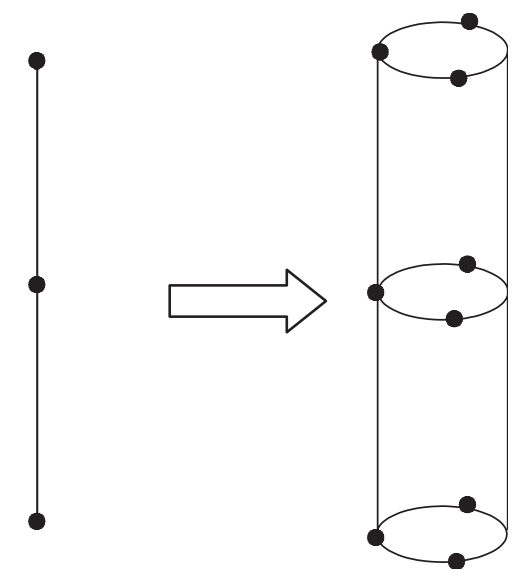

Figure 1. Generation of the surface of the fiber (and the surface of the hole containing the fiber) from user input nodes. 


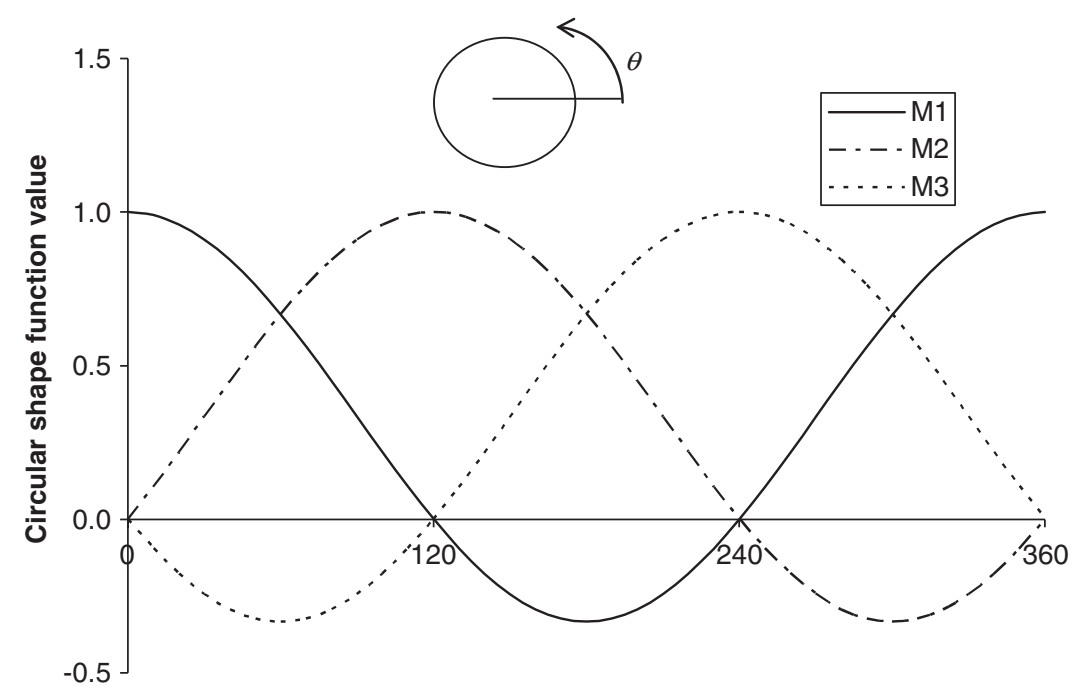

Theta (degree)

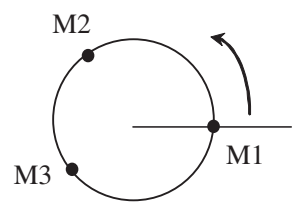

Figure 2. Values of three nodal circular shape functions about the fiber/hole.

Also, a modified shape function is used in the integration over the end of the hole to ensure continuity temperature and flux at the center of the end surface, which is given by

$$
\bar{M}^{\gamma}=a M^{\gamma}+b / 3, \quad \gamma=1,2,3
$$

with

$$
a=r / R \quad \text { and } \quad b=(R-r) / R
$$

where $R$ is the radius of the hole at the end, $r$ is the location of the integration (Gauss) point as it sweeps from $r=0$ to $r=R$ and $M^{\gamma}$ is the circular shape function defined in Equation (14).

Thus, using the above discretizations, dividing the body into $Q$ number of boundary elements and $P$ number of fiber elements, the boundary integral Equations (9) for the matrix with hole can be expressed as

$$
\begin{aligned}
C T^{N}(\xi, t)= & \sum_{q=1}^{Q}\left[\int_{S^{q}}\left[G_{\mathrm{O}}^{1}(x(\eta), t, \xi) L^{k}(\eta)\right] \mathrm{d} S^{q}(x(\eta))\right] q_{\mathrm{O}}^{N k} \\
& -\sum_{q=1}^{Q}\left[\int_{S^{q}}\left[F_{\mathrm{O}}^{1}(x(\eta), t, \xi) L^{k}(\eta)\right] \mathrm{d} S^{q}(x(\eta))\right] T_{\mathrm{O}}^{N k}
\end{aligned}
$$




$$
\begin{aligned}
& +\sum_{p=1}^{P}\left[\int_{S^{p}}\left[G_{\mathrm{H}}^{1}(x(\varsigma), t, \xi) N^{k}(\varsigma) M^{\gamma}(\theta)\right] \mathrm{d} S^{p}(x(\varsigma), \theta)\right] q_{\mathrm{H}}^{N k} \\
& -\sum_{p=1}^{P}\left[\int_{S^{p}}\left[F_{\mathrm{H}}^{1}(x(\varsigma), t, \xi) N^{k}(\varsigma) M^{\gamma}(\theta)\right] \mathrm{d} S^{p}(x(\varsigma), \theta)\right] T_{\mathrm{H}}^{N k}+R_{\mathrm{OH}}^{N-1}
\end{aligned}
$$

with

$$
\begin{aligned}
R_{\mathrm{OH}}^{N-1}= & \sum_{n=1}^{N-1}\left\{\sum_{q=1}^{Q}\left[\int_{S^{q}}\left[G_{\mathrm{O}}^{n}(x(\eta), t, \xi) L^{k}(\eta)\right] \mathrm{d} S^{q}(x(\eta))\right] q_{\mathrm{O}}^{n k}\right. \\
& -\sum_{q=1}^{Q}\left[\int_{S^{q}}\left[F_{\mathrm{O}}^{n}(x(\eta), t, \xi) L^{k}(\eta)\right] \mathrm{d} S^{q}(x(\eta))\right] T_{\mathrm{O}}^{n k} \\
& +\sum_{p=1}^{P}\left[\int_{S^{p}}\left[G_{\mathrm{H}}^{n}(x(\varsigma), t, \xi) N^{k}(\varsigma) M^{\gamma}(\theta)\right] \mathrm{d} S^{p}(x(\varsigma), \theta)\right] q_{\mathrm{H}}^{n k} \\
& \left.-\sum_{p=1}^{P}\left[\int_{S^{p}}\left[F_{\mathrm{H}}^{n}(x(\varsigma), t, \xi) N^{k}(\varsigma) M^{\gamma}(\theta)\right] \mathrm{d} S^{p}(x(\varsigma), \theta)\right] T_{\mathrm{H}}^{n k}\right\}
\end{aligned}
$$

In a similar fashion, the boundary integral equation (11) for the fiber can be expressed as

$$
\begin{aligned}
C_{\mathrm{F}} T^{N}(\xi, t)= & \sum_{p=1}^{P}\left[\int_{S^{p}}\left[G_{\mathrm{F}}^{1}(x(\varsigma), t, \xi) N^{k}(\varsigma) M^{\gamma}(\theta)\right] \mathrm{d} S^{p}(x(\varsigma), \theta)\right] q_{\mathrm{F}}^{N k} \\
& -\sum_{p=1}^{P}\left[\int_{S^{p}}\left[F_{\mathrm{F}}^{1}(x(\varsigma), t, \xi) N^{k}(\varsigma) M^{\gamma}(\theta)\right] \mathrm{d} S^{p}(x(\varsigma), \theta)\right] T_{\mathrm{F}}^{N k}+R_{\mathrm{F}}^{N-1}
\end{aligned}
$$

with

$$
\begin{aligned}
R_{\mathrm{F}}^{N-1}= & \sum_{n=1}^{N-1}\left\{\sum_{p=1}^{P}\left[\int_{S^{p}}\left[G_{\mathrm{F}}^{n}(x(\varsigma), t, \xi) N^{k}(\varsigma) M^{\gamma}(\theta)\right] \mathrm{d} S^{p}(x(\varsigma), \theta)\right] q_{\mathrm{F}}^{n k}\right. \\
& \left.-\sum_{p=1}^{P}\left[\int_{S^{p}}\left[F_{\mathrm{F}}^{n}(x(\varsigma), t, \xi) N^{k}(\varsigma) M^{\gamma}(\theta)\right] \mathrm{d} S^{p}(x(\varsigma), \theta)\right] T_{\mathrm{F}}^{n k}\right\}
\end{aligned}
$$

where $Q$ is the number of boundary elements on the outer surface of the composite matrix, $P$ is the number of fiber elements in the region, $L(\eta)$ represents a $2 \mathrm{D}$ shape function, $N(\varsigma)$ represents a one-dimensional (1D) shape function and $M^{\gamma}(\theta)$ are circular shape functions defined earlier. The superscript $k$ is used to denote the $k$ th node of the $P$ th and $Q$ th elements, respectively, of the matrix and the fiber, and, as mentioned previously, the subscripts $\mathrm{O}, \mathrm{H}$ and $\mathrm{F}$ are used to denote quantities on the outer boundary surface, surface of the hole and surface of the fiber, respectively. 


\subsection{Discussion on kernel singularities}

The fundamental solutions to the quasistatic problem contain singularities when the load point and the field point coincide. The same is true for analytically derived kernels $G^{n}$ and $F^{n}$, since these kernels are derived from the fundamental solutions. Series expansions of terms present in the evaluation functions can be used to deduce the level of singularities existing in the kernels.

A number of observations concerning the results of these expansions should be mentioned. First, as would be expected, $F^{1}$ has a stronger level of singularity than the corresponding $G^{1}$, since an additional derivative is involved in obtaining $F^{1}$ from $G^{1}$. Second, both kernel functions for the first time step could actually be re-written as a sum of steady-state and transient components. Then, the singularity is completely contained in the steady-state portion. This observation is critical in the numerical integration of the $F^{1}$ kernel. However, from a physical standpoint, this means that, at any time $t$, the closer one moves toward the load point, the closer the quasistatic response field corresponds to a steady-state field. Eventually, when the sampling and load point coincide, the quasistatic and the steady-state responses are indistinguishable. As a final note, after careful examination of the kernels presented in the Appendix, it is evident that the steady-state components in the kernels $G^{n}$ and $F^{n}$, with $n>1$, vanish. In that case, all that remains is a transient portion that contains no singularities. Thus, all singularities reside in the steady-state parts of the $G^{1}$ and $F^{1}$.

\section{ASSEMBLY AND SOLUTION}

After the temporal and spatial discretizations outlined in Equations (17)-(20) are complete, a system of algebraic equations can be developed to permit the solution of the quasistatic problem. This is accomplished by systematically writing the discretized equations at each node on the outer surface, on the surface of the hole and on the surface of the fibers. The resulting collocation process yields a global set of equations which can be expressed symbolically for the $N$ th time step in a suitable matrix form.

For the nodes on the outer surface and on the surface of the hole

$$
\left[G_{\mathrm{O}}^{1}\right]\left\{q^{N}\right\}-\left[F_{\mathrm{O}}^{1}\right]\left\{T^{N}\right\}+\left[G_{\mathrm{H}}^{1}\right]\left\{q^{N}\right\}-\left[F_{\mathrm{H}}^{1}\right]\left\{T^{N}\right\}+\left\{R_{\mathrm{OH}}^{N-1}\right\}=\{0\}
$$

where $q^{N}$ and $T^{N}$ are the nodal quantities, with the superscripts denoting the time step index, $R_{\mathrm{OH}}^{N-1}$ comprises of the convolution terms defined in Equation (18) before. Here, it should be pointed out that, during the above collocation process, an extension of the indirect equipotential techniques [20] has been utilized to determine the strongly singular terms in the diagonal blocks of the $F$ matrix. Alternatively, these could be regularized using the same procedure analytically [20]. A discussion of the application of these principles in the transient heat transfer can be found in Dargush and Banerjee [3].

For the matrix with hole, Equation (21) can be re-expressed for convenience as

$$
\left[G_{\mathrm{O}}^{1}\right]\left\{q_{\mathrm{O}}^{N}\right\}-\left[F_{\mathrm{O}}^{1}\right]\left\{T_{\mathrm{O}}^{N}\right\}+\left[G_{\mathrm{H}}^{1}\right]\left\{q_{\mathrm{H}}^{N}\right\}-\left[F_{\mathrm{H}}^{1}\right]\left\{T_{\mathrm{H}}^{N}\right\}=\left\{D_{\mathrm{OH}}^{N-1}\right\}
$$

where

$$
\left\{D_{\mathrm{OH}}^{N-1}\right\}=-\left\{R_{\mathrm{OH}}^{N-1}\right\}
$$


with $R_{\mathrm{OH}}^{N-1}$ defined in Equation (18), $D_{\mathrm{OH}}^{N-1}$ can be re-expressed as

$$
\left\{D_{\mathrm{OH}}^{N-1}\right\}=-\sum_{n=1}^{N-1}\left\{\left[G_{\mathrm{O}}^{n}\right]\left\{q_{\mathrm{O}}^{n}\right\}-\left[F_{\mathrm{O}}^{n}\right]\left\{T_{\mathrm{O}}^{n}\right\}+\left[G_{\mathrm{H}}^{n}\right]\left\{q_{\mathrm{H}}^{n}\right\}-\left[F_{\mathrm{H}}^{n}\right]\left\{T_{\mathrm{H}}^{n}\right\}\right\}
$$

Similarly, for the nodes on the fiber, we can write

$$
\left[G_{\mathrm{F}}^{1}\right]\left\{q_{\mathrm{F}}^{N}\right\}-\left[F_{\mathrm{F}}^{1}\right]\left\{T_{\mathrm{F}}^{N}\right\}=\left\{D_{\mathrm{F}}^{N-1}\right\}
$$

In Equation (24), the term $D_{\mathrm{F}}^{N-1}$ indicates convolution due to the fiber and is given by

$$
\left\{D_{\mathrm{F}}^{N-1}\right\}=-\left\{R_{\mathrm{F}}^{N-1}\right\}
$$

with $R_{\mathrm{F}}^{N-1}$ defined in Equation $(20), D_{\mathrm{F}}^{N-1}$ can be re-written as

$$
\left\{D_{\mathrm{F}}^{N-1}\right\}=-\sum_{n=1}^{N-1}\left\{\left[G_{\mathrm{F}}^{n}\right]\left\{q_{\mathrm{F}}^{n}\right\}-\left[F_{\mathrm{F}}^{n}\right]\left\{T_{\mathrm{F}}^{n}\right\}\right\}
$$

Using the compatibility condition at the interface between the matrix and the fiber, we can write

$$
\begin{aligned}
& \left\{T_{\mathrm{H}}\right\}=\left\{T_{\mathrm{F}}\right\} \\
& \left\{q_{\mathrm{H}}\right\}=-\left\{q_{\mathrm{F}}\right\}
\end{aligned}
$$

Substitution of Equations (28) and (29) into Equations (25) and (27) and re-arrangement yield

$$
\begin{aligned}
\left\{q_{\mathrm{H}}^{N}\right\} & =-[H]\left[F_{\mathrm{F}}^{1}\right]\left\{T_{\mathrm{H}}^{N}\right\}-[H]\left\{D_{\mathrm{F}}^{N-1}\right\} \\
\left\{D_{\mathrm{F}}^{N-1}\right\} & =\sum_{n=1}^{N-1}\left\{\left[G_{\mathrm{F}}^{n}\right]\left\{q_{\mathrm{H}}^{n}\right\}+\left[F_{\mathrm{F}}^{n}\right]\left\{T_{\mathrm{H}}^{n}\right\}\right\}
\end{aligned}
$$

where

$$
[H]=\left[G_{\mathrm{F}}^{1}\right]^{-1}
$$

Note, since the boundary integral equation for each fiber is independent of the equations for the other fibers, the inversion of $\left[G_{\mathrm{F}}^{1}\right]$ can be reduced to a number of smaller inversions, one corresponding to each fiber. Furthermore, when the composite matrix is divided into a multi-region model, the above fiber assembly can be performed for each region independently.

Equation (30) can now be back-substituted into Equation (22) to yield

$$
\left[G_{\mathrm{O}}^{1}\right]\left\{q_{\mathrm{O}}^{N}\right\}-\left[F_{\mathrm{O}}^{1}\right]\left\{T_{\mathrm{O}}^{N}\right\}-\left[F_{X}\right]\left\{T_{\mathrm{H}}^{N}\right\}=\left\{D_{\mathrm{OH}}^{N-1}\right\}+\left[G_{\mathrm{H}}^{1}\right][H]\left[D_{\mathrm{F}}^{N-1}\right]
$$

where

$$
\left[F_{X}\right]=\left[G_{\mathrm{H}}^{1}\right][H]\left[F_{\mathrm{F}}^{1}\right]+\left[F_{\mathrm{H}}^{1}\right]
$$

In a well-posed problem, at every point on the outer surface of the body the flux or the temperature or an inter-relationship is specified, and on the surface of the hole only the temperatures are retained. Therefore, the number of equations in the system is equal to the final number of unknowns. 
Then, at the final stage of the assembly process, Equation (32) can be expressed in the form

$$
\left[A^{1}\right]\left\{x^{N}\right\}=\left[B^{1}\right]\left\{y^{N}\right\}+\left\{D_{\mathrm{OH}}^{N-1}\right\}+\left[G_{\mathrm{H}}^{1}\right][H]\left\{D_{\mathrm{F}}^{N-1}\right\}
$$

in which $\left\{x^{N}\right\}$ are the unknown components of $\left\{T^{N}\right\}$ and $\left\{q^{N}\right\},\left\{y^{N}\right\}$ are the known components of $\left\{T^{N}\right\}$ and $\left\{q^{N}\right\}$ and $\left[A^{1}\right]$ and $\left[B^{1}\right]$ are associated coefficient matrices.

Note that the entire matrix $\left[F_{X}\right]$ becomes part of $\left[A^{1}\right]$ since all temperatures on the interfaces of the holes and fibers are unknown quantities.

\section{TREATMENT OF THE LEADING TIME STEP}

In the present discussion on the treatment of leading time step calculation, we first focus on the integrals to be evaluated on the boundary of the composite matrix.

For the leading time step calculation, the integrals on the outer boundary appearing in Equation (17) can be numerically evaluated to

$$
\int_{\Delta S^{q}}\left[G_{\mathrm{O}}^{1}(x(\eta), t, \xi) L^{k}(\eta) J(\eta) \mathrm{d} \eta\right] q_{\mathrm{O}}^{n k}
$$

and

$$
\int_{\Delta S^{q}}\left[F_{\mathrm{O}}^{1}(x(\eta), t, \xi) L^{k}(\eta) J(\eta) \mathrm{d} \eta\right] T_{\mathrm{O}}^{n k}
$$

where the superscript $k$ is used to represent the $k$ th node of the $Q$ th element, $L$ is a 2D shape function, $J$ is the Jacobian product and, $\eta$ is the intrinsic coordinate within the element.

It is evident from Equations (35) and (36) that the only approximation introduced in the evaluation of the above integrals is the nodal variation of quantities within the element via shape functions and the temporal variation over the step $\Delta t$.

In order to assist in the above endeavor, the following three distinct categories can be identified; (i) the point $\xi$ does not lie on the element to be integrated; (ii) the point $\xi$ lies on the element, but only non-singular or weakly singular integrals are involved; and (iii) the point $\xi$ lies on the element and the integral is strongly singular.

In practical problems involving many elements, it is evident that most of the integrals outlined in Equations (35) and (36) will be of category (i) variety. In this case, the integrand is always nonsingular, and standard Gaussian quadrature formulas can be employed. However, sophisticated error control routines are needed to minimize the computational effort for a certain level of accuracy. This non-singular integration is the most expensive part of a boundary element analysis and, consequently, must be optimized to achieve an efficient solution. These are discussed extensively in BEM literature and were first introduced by Lachat and Watson [25], and subsequently modified in the works of Banerjee et al. [26] and Mustoe [27].

Turning to the next category (ii), one finds that again Gaussian quadrature is applicable; however, a somewhat modified scheme must be utilized to evaluate the weakly singular integrals. This is accomplished through element sub-segmentation about the singular point so that the product of shape function, Jacobian and the kernel function remains well behaved. As mentioned before, the strongly singular integral can be evaluated indirectly using a modified equipotential technique $[3,20]$. 
Next, we focus on the integrals to be evaluated on the surface of the hole and fiber elements. The complexity of the transient kernel functions prohibits an analytical integration about the circumference of the fiber proposed by Banerjee and Henry [7] and Henry and Banerjee [6]. Therefore, numerical integration must be performed in both the longitudinal and the circumferential directions. As an example, the integrals about the surface of the $P$ th fiber can be expressed as

$$
\begin{aligned}
& \int_{\Delta S^{p}}\left[G_{\mathrm{F}}^{1}(x(\varsigma), t, \xi) N^{k}(\varsigma) M^{\gamma}(\theta) \mathrm{d} S^{p}(x(\varsigma), \theta)\right] q_{\mathrm{F}}^{n k} \\
& \int_{\Delta S^{p}}\left[F_{\mathrm{F}}^{1}(x(\varsigma), t, \xi) N^{k}(\varsigma) M^{\gamma}(\theta) \mathrm{d} S^{p}(x(\varsigma), \theta)\right] T_{\mathrm{F}}^{n k}
\end{aligned}
$$

In Equations (37) and (38), the superscript $k$ is used to represent the $k$ th node of the $P$ th element, $N(\varsigma)$ is a $1 \mathrm{D}$ shape function, and $\varsigma$ is the intrinsic coordinate along the length of the fiber element and along the radial direction for the end disk integration.

For the evaluation of the integrals outlined in Equations (37) and (38), an efficient integration scheme is adopted in which 1D Gaussian integration formulas are applied independently in the longitudinal and circumferential directions. This allows sub-segmentation and mapping similar to that explained for the outer boundary integration explained earlier, as well as the order of the Gaussian formulas to vary independently in the two directions so that the accuracy and cost of the integration can be optimized.

To perform the above numerical integration, a local cylindrical coordinate system $(r, \theta, z)$ is constructed, with the center of the coordinate system coinciding with the center of the fiber and the $z$-axis aligned with the centerline of the fiber. With respect to this coordinate system, let $\left(r_{x}, \theta_{x}, z_{x}\right)$ and $\left(r_{\xi}, \theta_{\xi}, z_{\xi}\right)$ be the coordinates of the load point and the field point, respectively. Then, after the numerical integration in the circumferential direction, Equations (37) and (38) can be symbolically expressed as

$$
\begin{aligned}
& \int_{\Delta S^{p}}\left[G^{\gamma}(z(\varsigma), t, \xi) N^{k}(\varsigma) J(\varsigma) \mathrm{d} \varsigma\right] q_{\mathrm{F}}^{n k} \\
& \int_{\Delta S^{p}}\left[F^{\gamma}(z(\varsigma), t, \xi) N^{k}(\varsigma)(\varsigma) \mathrm{d} \varsigma\right] T_{\mathrm{F}}^{n k}
\end{aligned}
$$

where for the positions of the three circumferential nodes (see Figure 3), $G^{\gamma}$ and $F^{\gamma}$ with $\gamma=1,2,3$ (corresponding to the three values of $\theta_{\xi}=0,+2 \pi / 3$ and $-2 \pi / 3$ ) are given by

$$
\begin{aligned}
G^{1} & =\left[\frac{1}{3} \sum_{i} W_{i} G_{i}^{n} \mathrm{~d} \theta_{x_{i}}+\left(\frac{2}{3} \cos \theta_{\xi}\right) \sum_{i} W_{i} G_{i}^{n} \cos \theta_{x i}\right] R^{2} \\
G^{2} & =\left[\frac{1}{3} \sum_{i} W_{i} G_{i}^{n} \mathrm{~d} \theta_{x_{i}}+\left(\frac{\sqrt{3}}{3} \sin \theta_{\xi}-\frac{1}{3} \cos \theta_{\xi}\right) \sum_{i} W_{i} G_{i}^{n} \cos \theta_{x i}\right] R^{2} \\
G^{3} & =\left[\frac{1}{3} \sum_{i} W_{i} G_{i}^{n} \mathrm{~d} \theta_{x_{i}}+\left(-\frac{\sqrt{3}}{3} \cos \theta_{\xi}-\frac{1}{3} \sin \theta_{\xi}\right) \sum_{i} W_{i} G_{i}^{n} \cos \theta_{x i}\right] R^{2}
\end{aligned}
$$




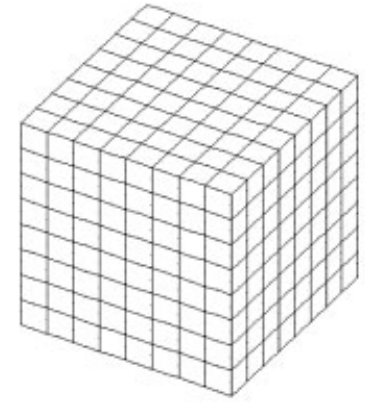

Outside boundary mesh

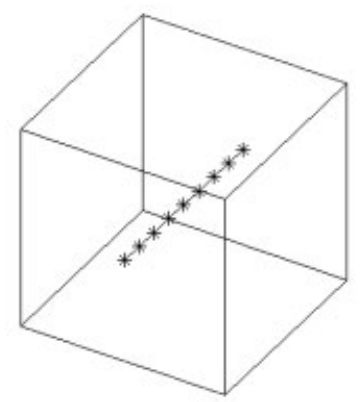

Cube with one fiber, fiber element

Figure 3. Cube with a single fiber, BEM mesh used in 3D fiber element formulation.

$$
\begin{aligned}
F^{1} & =\left[\frac{1}{3} \sum_{i} W_{i} F_{i}^{n} \mathrm{~d} \theta_{x_{i}}+\left(\frac{2}{3} \cos \theta_{\xi}\right) \sum_{i} W_{i} F_{i}^{n} \cos \theta_{x i}\right] R^{2} \\
F^{2} & =\left[\frac{1}{3} \sum_{i} W_{i} F_{i}^{n} \mathrm{~d} \theta_{x_{i}}+\left(\frac{\sqrt{3}}{3} \sin \theta_{\xi}-\frac{1}{3} \cos \theta_{\xi}\right) \sum_{i} W_{i} F_{i}^{n} \cos \theta_{x i}\right] R^{2} \\
F^{3} & =\left[\frac{1}{3} \sum_{i} W_{i} F_{i}^{n} \mathrm{~d} \theta_{x_{i}}+\left(-\frac{\sqrt{3}}{3} \cos \theta_{\xi}-\frac{1}{3} \sin \theta_{\xi}\right) \sum_{i} W_{i} F_{i}^{n} \cos \theta_{x i}\right] R^{2}
\end{aligned}
$$

In the above expressions, $G^{n}$ and $F^{n}$ are transient kernels mentioned earlier, $W_{i}$ represents the $i$ th Gauss point weight and $R$ is the radius of the fiber/hole.

Using the same expressions outlined in Equations (41) and (42) for circumferential integration about the fiber, the integration of the end disk can be accomplished numerically with respect to $r$ (see Equation (16)).

\section{TREATMENT OF CONVOLUTION INTEGRALS}

\subsection{Introduction}

In this section, the detailed treatment of the convolution terms $R^{N-1}$ is presented for the evaluation of the convolution integrals on the outer boundary, on the surface of hole and on the surface of the fibers.

5.2. Treatment of $R^{N-1}$ via accurate integration-based convolution algorithm for the integrals on the surface of the hole and on the surface of the fibers

In this algorithm, the storage is eliminated by recalculating all the coefficients of the past time steps to form the summation $R^{N-1}$ at every time step as a totally new and renumbered set of calculations $[23,24]$.

Similar to the procedure outlined for the case of leading time step calculation, using appropriate nodal shape functions and the Jacobian product involved in the mapping process [20], the integrals 
can be numerically evaluated up to the desired accuracy. In this process, a time-embedded distance criterion has been used to allow for the fact that distant elements (both in terms of space and time) require smaller number of Gauss points. These are more fully developed for the matrix in the next section.

\subsection{Treatment of $R^{N-1}$ via the new fast convolution algorithm for integrals on the outer boundary surface}

This section entirely focuses on the new fast convolution algorithm used in this work for the evaluation of the convolution integrals on the outer boundary.

To explain the above procedure, first, the typical convolution integrals on the outer boundary are presented as

$$
\int_{\Delta S^{q}}\left[G_{\mathrm{O}}^{n}(x(\eta), t, \xi) L^{k}(\eta) J(\eta) \mathrm{d} \eta\right] q_{\mathrm{O}}^{n k}
$$

and

$$
\int_{\Delta S^{q}}\left[F_{\mathrm{O}}^{n}(x(\eta), t, \xi) L^{k}(\eta) J(\eta) \mathrm{d} \eta\right] T_{\mathrm{O}}^{n k}
$$

Using this new algorithm, the integrals appearing in Equations (43) and (44) are further adjusted and subdivided into different distinct categories based on time-embedded distance criteria. A different order of Gauss points is used for the evaluation of each type of integrals based on their effects on the leading time step. We also use temporally and spatially integrated average values of temperature and flux for all steps from $n=1$ to $n=N-1$, for an element, instead of using functional variations using all nodes of an element to represent the total past effect for the righthand side calculation, thus preserving the energy balance as expressed by the original exact forms contained in Equations (1) and (2). This significantly reduces the computing time and cost while preserving the energy balance of the discretized boundary integral equation.

In order to illustrate the time-embedded distance criterion used in this new algorithm, for the $N$ th time step, let us define the time criterion $N_{T}$ as the number of time intervals from $n=1$ to $n=N-1$. Also let us define the distance criterion as $N_{s}=r / l$, where $r$ is the distance from the field point to the center of the element to be integrated and $l$ is the corresponding element length. The time-embedded distance criterion used in the present work is based on the product $N_{T} N_{S}$ $[23,24]$.

Based on the above guidelines, we could choose to associate $L^{k}(\eta)$ with $q(x)$ and $T(x)$ and define

$$
\begin{aligned}
\Delta S^{q} \bar{q}^{n k} & =\int_{S^{q}}\left[L^{k}(\eta) J(\eta) \mathrm{d} \eta\right] q^{n k} \\
\Delta S^{q} \bar{T}^{n k} & =\int_{\Delta s^{q}}\left[L^{k}(\eta) J(\eta) \mathrm{d} \eta\right] T^{n k}
\end{aligned}
$$

where

$$
\Delta S^{q}=\int_{\Delta s^{q}} J(\eta) \mathrm{d} \eta
$$


Then we could write integrals in Equations (43) and (44) as

$$
\begin{aligned}
\bar{q}^{n k} \int_{\Delta s^{q}} G^{n}(x, \xi, t) \mathrm{d} S^{q} & =\bar{q}^{n k} \int_{\Delta s^{q}} G^{n}(x(\eta), \xi, t) J(\eta) \mathrm{d} \eta \\
\bar{T}^{n k} \int_{\Delta s^{q}} F^{n}(x, \xi, t) \mathrm{d} S^{q} & =\bar{T}^{n k} \int_{\Delta s^{q}} F^{n}(x(\eta), \xi, t) J(\eta) \mathrm{d} \eta
\end{aligned}
$$

We use the integrals outlined in Equations (48) and (49) in close proximity and early convolution times according to the guidelines described above.

As time progresses, the effects of early time values have progressively less influence on the current time steps. Therefore, for these steps, the integrals on the right-hand sides of Equations (48) and (49) can be further approximated to

$$
\begin{aligned}
& \bar{q}^{n k} G^{n}\left(x^{q}, \xi, t\right) \Delta S^{q} \\
& \bar{T}^{n k} F^{n}\left(x^{q}, \xi, t\right) \Delta S^{q}
\end{aligned}
$$

It should be noted that, although these contributions are small, they cannot be neglected because their total sum retains the balance of energy and without that the steady-state solution could never be reached.

\section{NUMERICAL ACCURACY}

\subsection{Cube with a single fiber}

To test the accuracy of the present transient BEM fiber element formulation, in this first verification example, the transient heat conduction through a 3D unit cube with a single fiber is studied. The radius of the fiber is 0.1 . The cube is initially at a temperature of $0^{\circ}$. Then, a uniform flux of 100.0 is suddenly applied to a side of the cube parallel to the fibers, while the opposite side of the cube is maintained at a temperature of $0^{\circ}$. All other faces of the cube are insulated. For comparison, a 2D two-region BEM analysis is also carried out for the same problem. Note that, for the representation of the circumference, eight quadratic elements are needed leading to 16 nodes, whereas in the fiber element BEM, we are using analytical representation of the geometry and only three nodes for functional variation.

The BEM mesh used in this problem for the 3D fiber element formulation is shown in Figure 3 and the corresponding multi-region 2D BEM meshes are shown in Figure 4. The outside boundary mesh for the fiber element formulation consists of 384 eight-noded quadratic elements (64 elements on each face), whereas one three-noded quadratic element is used to model the fiber. The 2D BEM mesh uses three-noded quadratic elements on the boundary and on the surface of the hole and fiber. It should be noted that although about four quadratic elements per face of the cube could have been adequate for the current problem, we wish to solve several more complex problems of multiple fibers by retaining the same boundary mesh and changing only the mesh for the fibers.

To carry out the present study, the following material properties in consistent units are assumed for the matrix and the fiber, matrix: $\rho c_{\varepsilon}=1.0, k=1.0$; fiber: $\rho c_{\varepsilon}=1.0, k=20.0$, where $\rho$ is the density, $c_{\varepsilon}$ is the specific heat and $k$ is the conductivity. 


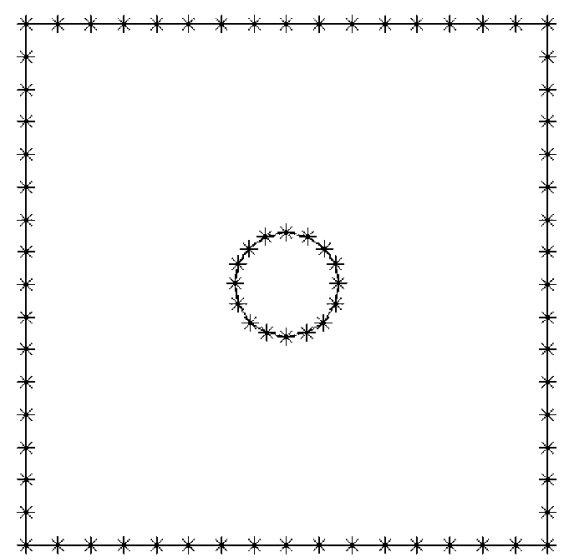

Figure 4. Cube with a single fiber, two-region 2D BEM mesh.

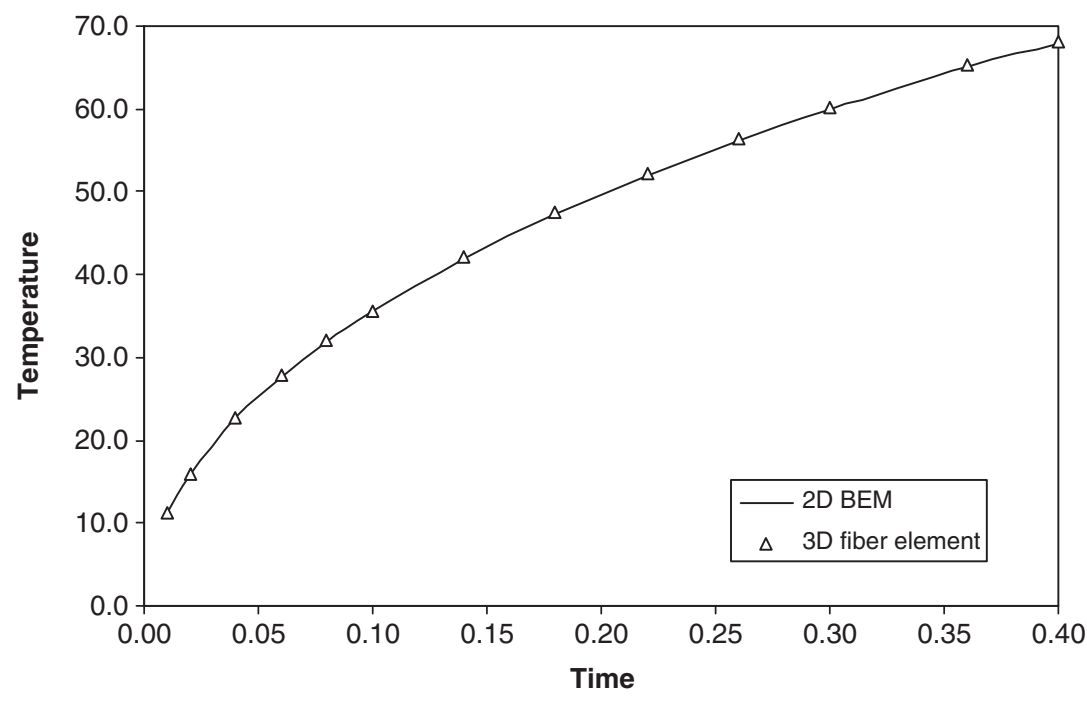

Figure 5. Cube with a single fiber, temperature variation with time.

The variation of temperature with time at a point located in the middle of the face where flux is applied is presented in Figure 5 using 3D fiber element composite formulation as well as using a two-region BEM approach. The solutions presented are obtained using 40 time increments with a time step size of 0.01 . The 3D fiber element composite solutions are seen to be in excellent agreement with the corresponding 2D BEM results even at early times.

Also, a comparison between the numerical results of temperature distribution along the face of the cube subjected to flux (at time 0.40) obtained using the fiber element formulation and those two-region BEM is presented in Table I. The composite solution is once again in very good agreement with the $2 \mathrm{D}$ BEM results. 
Table I. Cube with a single fiber, temperature profile along the side of cube at time 0.40 , comparison of results.

\begin{tabular}{lcc}
\hline $\begin{array}{l}\text { Distance from } \\
\text { side of cube }\end{array}$ & $\begin{array}{c}\text { Temperature } \\
\text { (2D BEM) }\end{array}$ & $\begin{array}{c}\text { Temperature } \\
\text { (3D fiber element) }\end{array}$ \\
\hline 0.0 & 68.77 & 68.66 \\
0.125 & 68.50 & 68.61 \\
0.25 & 68.24 & 68.38 \\
0.375 & 68.02 & 68.19 \\
0.50 & 67.92 & 68.10 \\
0.625 & 68.02 & 68.16 \\
0.75 & 68.24 & 68.34 \\
0.875 & 68.49 & 68.55 \\
1.0 & 68.57 & 68.60 \\
\hline
\end{tabular}

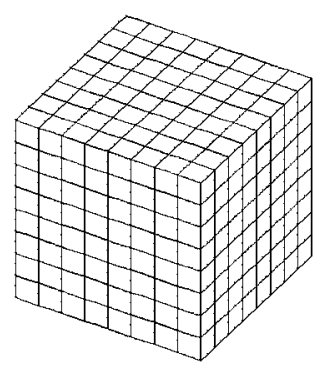

Outside boundary mesh

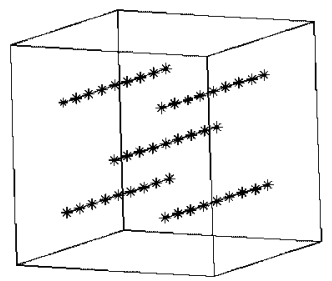

Cube with five fibers, fiber elements

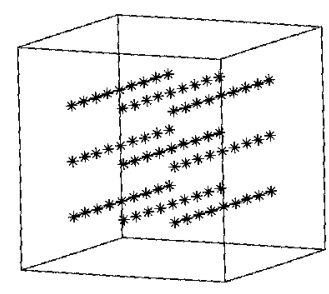

Cube with nine fibers, fiber elements

Figure 6. Cube with multiple fibers, BEM meshes for fiber element formulation.

\subsection{Cube with multiple fibers}

Next we examine the transient heat conduction in a 3D unit cube with multiple fibers. For this study, the 3D unit cube described in Example 6.1 is modeled with five and nine fibers, respectively. The corresponding BEM mesh details are shown in Figures 6 and 7, respectively, for the 3D fiber element and 2D multi-region BEM formulation. As before, initially the cube is assumed to be in thermal equilibrium at a temperature of $0^{\circ}$. Then, suddenly, a flux of 100.0 is applied at a face parallel to the fibers. The opposite face is maintained at a temperature of $0^{\circ}$. All the other faces are assumed to be insulated. As before, the conductivity of the fibers is assumed to be 20 times 


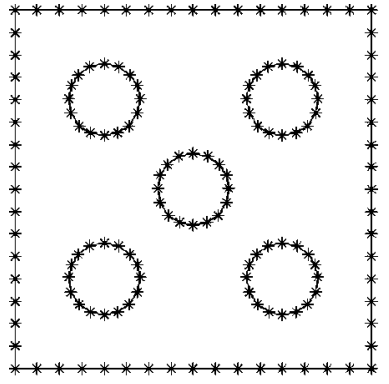

Cube with five fibers (six-region BEM mesh)

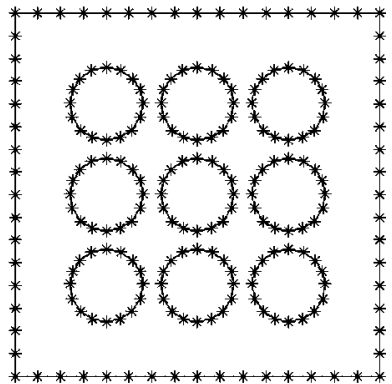

Cube with nine fibers (ten-region BEM mesh)

Figure 7. Cube with multiple fibers, 2D multi-region BEM meshes.

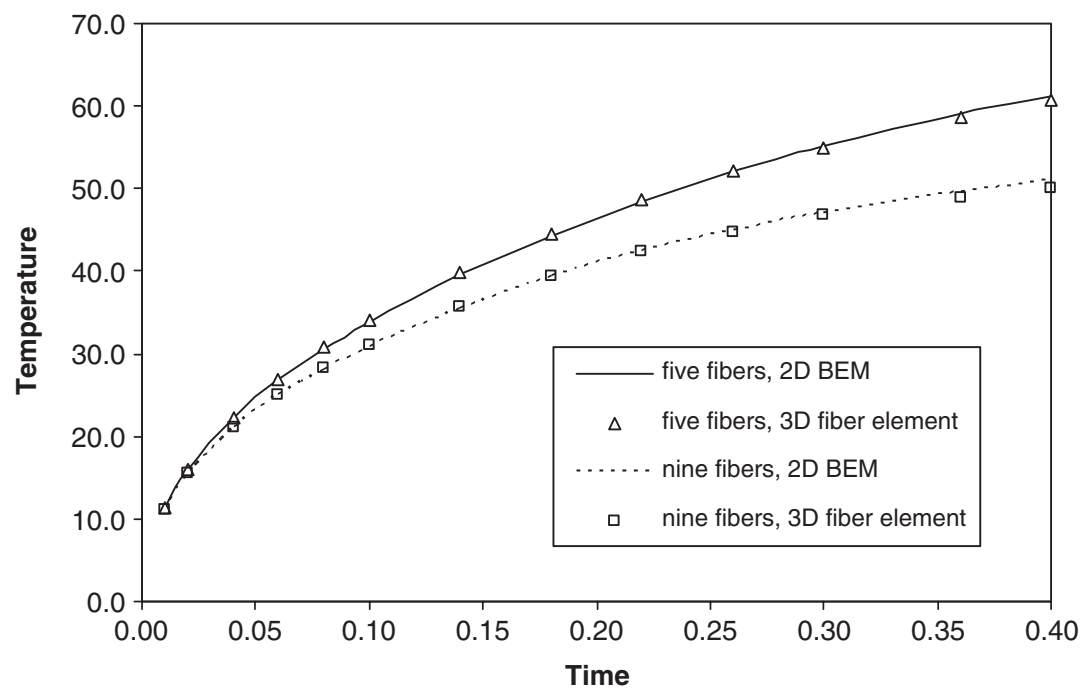

Figure 8. Cube with multiple fibers, temperature variations with time.

the conductivity of the matrix and the product of the specific heat and density is assumed to be unity for both the matrix and the fibers.

Again, the analyses are carried out using 40 time increments and a time step size of 0.01 . The resulting temperature variations with time at the middle of the face subjected to flux are shown in Figure 8 for the two different fiber arrangements. The numerical results of the fiber element formulation are seen to be in close agreement with the corresponding 2D multi-region BEM solutions. However, slight deviation near the steady state, as observed from the plot, can be attributed to the fact that we are using fibers of larger diameter than originally intended because we deliberately wanted the most accurate multi-region BEM results via full modeling. As the fibers become smaller in cross section, the regular BEM (two-region modeling) becomes more inaccurate. 


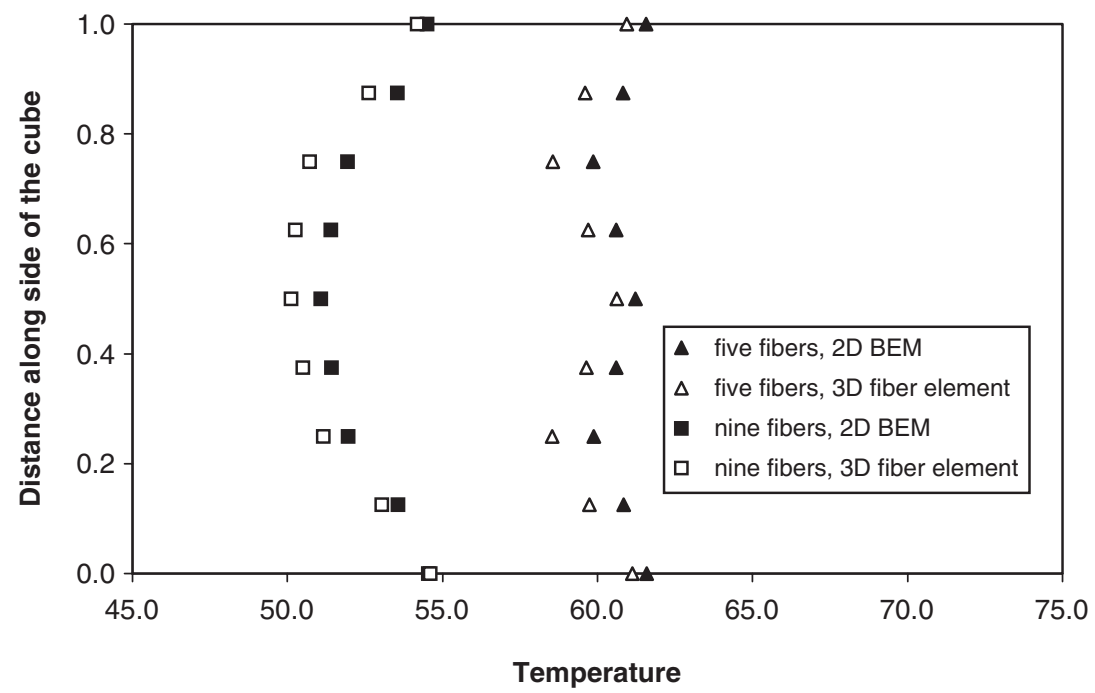

Figure 9. Cube with multiple fibers, temperature profile along the side of the cube at time 0.40 .

The temperature profile at time 0.40 along the side of the cube subjected to flux is depicted in Figure 9. The composite solutions are seen to be in close agreement with the $2 \mathrm{D}$ multi-region BEM results. Here, the effect of the number of fibers on the temperature profile is of particular interest. As the number of fibers is increased, the overall conductivity of the cube is increased. Hence, the heat from the applied flux is carried away (to the face which is maintained at $0^{\circ}$ ) at a higher rate, resulting in lower temperatures. Also note that the local temperature minimums in the temperature profiles are associated with the close proximity of the fibers near the flux boundary.

\section{EXAMPLE OF APPLICATION}

\subsection{Transient heat conduction analysis of a turbine blade}

After verifying the accuracy of the current fiber element composite formulation for transient BEM analysis, in an attempt to demonstrate the application and usefulness of the developed analysis, we study the transient heat conduction through a turbine blade. A boundary element discretization of a turbine blade is shown in Figure 10. Half symmetry is employed in this model which consists of 92 quadratic elements on the outer boundary. The model is $58.2 \mathrm{~mm}$ long, $13.79 \mathrm{~mm}$ wide, the radius of the base is $6.95 \mathrm{~mm}$ and the tip is $1.98 \mathrm{~mm}$ (from the plane of symmetry) thick at the largest point. The blade also consists of 26 fibers (13 fibers per side) running from the tip of the blade to half way through the base, as shown in Figure 10. The radius of each fiber is $0.15 \mathrm{~mm}$. Seven quadratic fiber elements are used to model each fiber, and thus the total number of fiber elements used in this problem is 92 .

A transient heat conduction analysis is first carried out on a homogeneous blade (blade with no fibers) with a conductivity of $k=0.0216 \mathrm{~W} / \mathrm{mm}^{\circ} \mathrm{C}$. The blade initially rests in thermodynamic equilibrium at zero temperature. Then, a gas at a temperature of $1200^{\circ} \mathrm{C}$ is assumed to flow over 


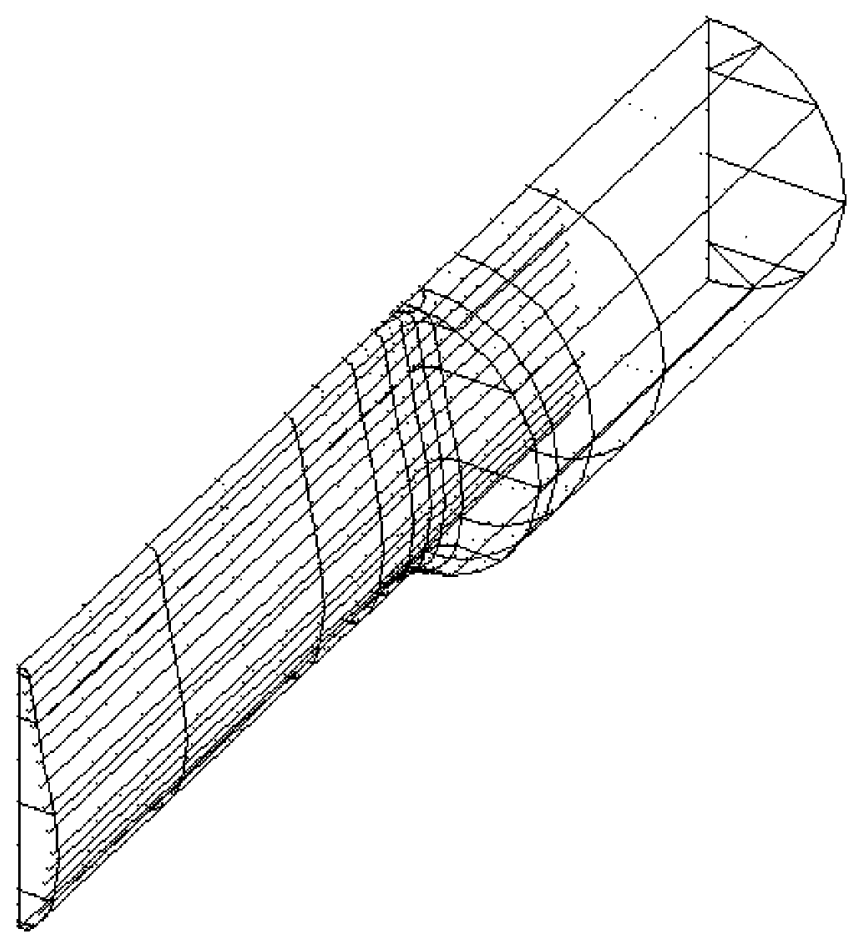

Figure 10. Turbine blade with fiber elements, BEM mesh.

the blade, while a gas at a temperature of $500^{\circ} \mathrm{C}$ surrounds the base of the blade. The difference between the surface temperature and the ambient temperature is assumed to be linearly related to the heat flux via a film coefficient which can be expressed as

$$
Q=-h\left(T_{\mathrm{a}}-T\right)
$$

where $Q$ is the heat flux, $T$ and $T_{\mathrm{a}}$ are the surface and ambient temperatures, respectively. $Q$ is positive if heat is leaving the body and negative if heat is entering the body. Using this type of boundary condition, $Q$ is not specified, but its value is determined from $h, T_{\mathrm{a}}$ and the temperature at the surface $T$. In this problem, at the leading edge of the blade, the film coefficient $h=0.003395 \mathrm{~W} / \mathrm{mm}^{2}{ }^{\circ} \mathrm{C}$ and tapers off to $0.00064 \mathrm{~W} / \mathrm{mm}^{2}{ }^{\circ} \mathrm{C}$ at the trailing edge. At the base of the blade it is assumed that the the film coefficient $h=0.00005 \mathrm{~W} / \mathrm{mm}^{2}{ }^{\circ} \mathrm{C}$. The analysis is carried out using eight time increments using a time-step size of $1.0 \mathrm{~s}$.

In order to compare the difference in the nature of heat conduction between a homogeneous blade and a fiber-reinforced blade, the blade is re-analyzed, this time with fibers as shown in Figure 10. The conductivity of the fibers is assumed to be 100 times the conductivity of the blade material.

The resulting temperature distributions along a vertical cross section at the center of the tip of the blade are shown in Figure 11 for the above two cases at times 2.0 and $8.0 \mathrm{~s}$. Even at time $2.0 \mathrm{~s}$, the temperature at the tip of the fiber-reinforced blade appears to be significantly lower than that 


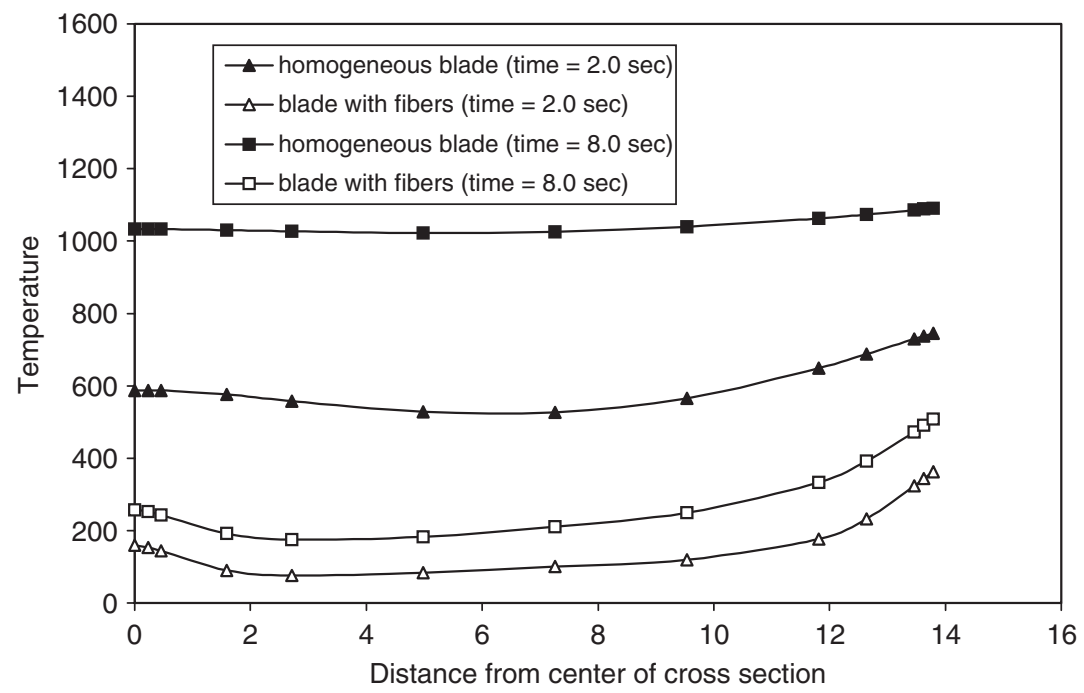

Figure 11. Temperature distribution at the tip of the turbine blade.

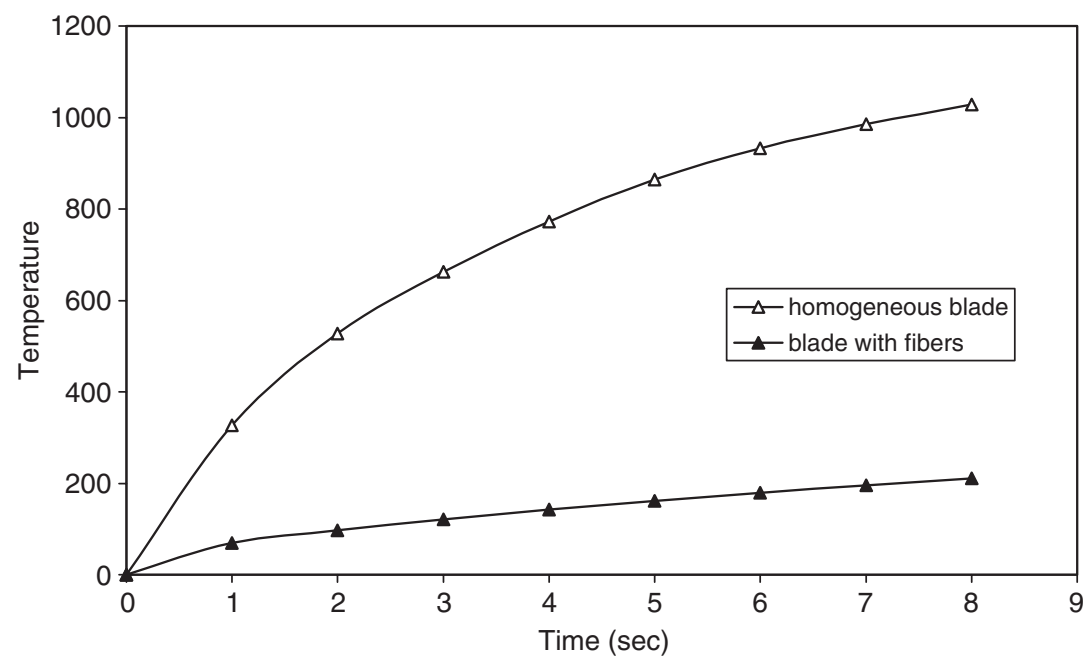

Figure 12. Temperature increase with time at the tip of the turbine blade.

of the homogeneous blade. The increase in temperature with time for the two cases is plotted in Figure 12 for a point on the tip surface. As expected, the increase in temperature with time for the fiber-reinforced blade is much slower, as opposed to significant rise in temperature between time 1.0 and $8.0 \mathrm{~s}$ for the homogeneous blade. This is due to the fact that the higher conductivity of the fibers increases the heat flow through the blade. Hence, the heat at the tip of the blade is carried toward the base faster than that in the homogeneous blade. The effect of fibers tends to lower the temperature quicker near the tip as compared with the homogeneous blade. 


\section{DISCUSSION AND CONCLUSIONS}

A new BEM development suitable for application to any multi-scale analysis of transient problems of heat conduction is presented in this paper. The analysis has been applied to a number of test problems to indicate that the results are sufficiently accurate to merit its use to practical problems. Finally, an application to the transient heat conduction of a turbine blade with and without fibers is shown to indicate the usefulness of the developed formulation.

The entire focus of our paper was on very small diameter fibers; for larger arbitrary rigid or flexible inclusions, we would recommend the use of full BEM modeling. For a very large number of small diameter inclusions (fibers) the more important component in the development of the analysis is the analytical representation of the geometry. The functional variation which can take into account a rigid mode, a compressible mode and a flexible mode is good enough. This has been achieved with the three-term Fourier expansion. Recently, we have extended the above analyses to inelastic deformation of solids, which unfortunately requires five terms but still remains reasonably efficient, particularly in terms of the total data preparation as well as computing cost perspective.

\section{APPENDIX}

This section provides the details of all the kernel functions used in the present transient heat conduction formulation, based on a continuous source fundamental solution. As a result, the following relationships must be used to determine the proper form of the functions required in the boundary element discretization. That is,

$$
\begin{aligned}
& G^{n}(x, \xi)=G(x, \xi, n \Delta t) \quad \text { for } n=1 \\
& G^{n}(x, \xi)=G(x, \xi, n \Delta t)-G(x, \xi,(n-1) \Delta t) \quad \text { for } n>1
\end{aligned}
$$

with similar expressions holding for the remaining kernels. In the specification of the kernels below, the arguments $(x, \xi, t)$ are assumed.

The temperature kernel is given by

$$
G=\frac{1}{4 \pi k r} \operatorname{erfc}\left(\frac{\eta}{2}\right)
$$

whereas for the flux kernel

$$
F=\frac{1}{4 \pi r^{2}}\left(\frac{y_{i} n_{i}}{r}\right)\left[1-h_{1}(\eta)\right]
$$

where

$$
\begin{aligned}
\operatorname{erfc}(z) & =1-\operatorname{erf}(z) \\
\operatorname{erf}(z) & =\frac{2}{\sqrt{\pi}} \int_{0}^{z} \mathrm{e}^{-x^{2}} \mathrm{~d} x \\
h_{1} & =\operatorname{erf}\left(\frac{\eta}{2}\right)-\frac{\eta}{\sqrt{\pi}} \mathrm{e}^{-\eta^{2} 4}
\end{aligned}
$$


The error functions utilized in the above expressions can be evaluated using common series approximations as outlined in Abramowitz and Stegun [28].

In the above, $x_{i}$ are the coordinates of integration point, $\xi_{i}$ are the coordinates of the field point.

$$
\begin{array}{cc}
y_{i}=x_{i}-\xi_{i}, \quad r^{2}=y_{i} y_{i} \\
\eta=\frac{r}{(c t)^{1 / 2}}, \quad c=\frac{k}{\rho c_{\varepsilon}}
\end{array}
$$

where $k$ is the conductivity, $\rho$ is the density and $c_{\varepsilon}$ is the specific heat.

\section{ACKNOWLEDGEMENTS}

We are deeply indebted to the BEST Corporation of Getzville, New York, for making available several blocks of the General Purpose Boundary Element Software Technology (GPBEST) system for this development.

\section{REFERENCES}

1. Banerjee PK, Butterfield R. The problem of pile cap-pile group interaction. Geotechniques 1971; 21:135-142.

2. Barone MR, Caulk DA. Special boundary integral equations for approximate solution of potential problems in three-dimensional regions with slender cavities of circular cross section. IMA Journal of Applied Mathematics 1985; 35:311-325.

3. Dargush GF, Banerjee PK. Application of boundary element method to transient heat conduction. International Journal for Numerical Methods in Engineering 1991; 31:1231-1248.

4. Rizzo FJ, Shippy DJ. A boundary integral approach to potential and elasticity problems for axisymmetric bodies with arbitrary boundary conditions. Mechanics Research Communications 1979; 6:99-103.

5. Wang HC, Banerjee PK. Multi-domain general axisymmetric analysis by BEM. International Journal for Numerical Methods in Engineering 1989; 28:2065-2083.

6. Henry DP, Banerjee PK. Elastic analysis of three-dimensional solids with small holes by BEM. International Journal for Numerical Methods in Engineering 1991; 31:369-384.

7. Banerjee PK, Henry DP. Elastic analysis of three-dimensional solids with fiber inclusions by BEM. International Journal of Solids and Structures 1992; 29:2423-2440.

8. Barone MR, Caulk DA. A new method for thermal analysis of die casting. Journal of Heat Transfer (ASME) 1993; 115:284-293.

9. Liu YL, Nishimura N, Otani Y, Takahashi T, Chen XL, Munakata H. A fast boundary element method for the analysis of fiber-reinforced composites based on a rigid inclusion model. Journal of Applied Mechanics (ASME) 2005; 72:115-128.

10. Nishimura N, Liu YL. Thermal analysis of carbon-nanotube composites using a rigid-line inclusion model by the boundary integral equation method. Computational Mechanics 2004; 35:1-10.

11. Banerjee PK, Henry DP, Dargush GF. Micromechanical studies of composites by BEM. Enhancing Analysis Techniques for Composite Materials. Proceedings of ASME Winter Annual Meeting, Atlanta, GA, 1991.

12. Rizzo FJ, Shippy DJ. An advanced boundary integral equation for three-dimensional thermoelasticity. International Journal for Numerical Methods in Engineering 1977; 11:1753-1768.

13. Henry DP, Banerjee PK. A new boundary element formulation for two- and three-dimensional thermoelasticity using particular integrals. International Journal for Numerical Methods in Engineering 1988; 26:2061-2078.

14. Yang MT, Park KH, Banerjee PK. 2D and 3D transient heat conduction analysis by BEM via particular integrals. Computer Methods in Applied Mechanics and Engineering 2002; 191:1701-1722.

15. Tomlin GR. Numerical analysis of continuum problems in zoned anisotropic media. Ph.D. Dissertation, Southampton University, U.K., 1973.

16. Chang YP, Kang CS, Chen DJ. The use of fundamental Green functions for the solution of problems of heat conduction in anisotropic media. International Journal of Heat and Mass Transfer 1973; 16:1905-1918. 
17. Banerjee PK, Butterfield R, Tomlin GR. Boundary element methods for two-dimensional problems of transient groundwater flow. International Journal for Numerical and Analytical Methods in Geomechanics 1981; 5:15-31.

18. Dargush GF. Boundary element methods for the analogous problems of thermomechanics and soil consolidation. Ph.D. Dissertation, State University of New York at Buffalo, Buffalo, NY, 1987.

19. Dargush GF, Banerjee PK. Advanced development of the boundary element method for steady state heat conduction. International Journal for Numerical Methods in Engineering 1989; 28:2123-2142.

20. Banerjee PK. The Boundary Element Methods in Engineering Science. McGraw-Hill: London, 1994.

21. Zabaras N, Mukherjee S. An analysis of solidification problems by the BEM. International Journal for Numerical Methods in Engineering 1987; 24:1879-1900.

22. Zabaras N, Mukherjee S, Richmond O. An analysis of inverse heat transfer problems with phase change using an integral method. Journal of Heat Transfer 1988; 110:554-561.

23. Chatterjee J, Ma F, Henry DP, Banerjee PK. Two- and three-dimensional transient heat conduction and thermoelastic analyses by BEM via efficient time convolution. Computer Methods in Applied Mechanics and Engineering 2007; 196:2828-2838.

24. Ma F, Chatterjee J, Banerjee PK. A new fast convolution algorithm in BEM for two- and three-dimensional linear soil consolidation analysis. International Journal of Geomechanics (ASCE) 2007; 7(3):236-249.

25. Lachat JC, Watson JO. Effective numerical treatment of boundary integral equations: a formulation for threedimensional elastostatics. International Journal for Numerical Methods in Engineering 1976; 10:991-1005.

26. Banerjee PK, Wilson RB, Miller NM. Advanced elastic and inelastic three-dimensional analysis of gas turbine engine structures by BEM. International Journal for Numerical Methods in Engineering 1988; 26:393-411.

27. Mustoe GCW. Advanced integration schemes over boundary elements and volume cells for two- and threedimensional non-linear analysis. Developments in Boundary Element Methods. Elsevier: London, 1984.

28. Abramowitz M, Stegun IA. Handbook of Mathematical Functions. Dover: New York, 1974. 